\title{
4 Measures of Regional Inequality for Small Countries
}

\author{
Boris A. Portnov ${ }^{1}$ and Daniel Felsenstein ${ }^{2}$ \\ ${ }^{1}$ Department of Natural Resources and Environmental Management, University \\ of Haifa, Israel \\ 2 Department of Geography, Hebrew University of Jerusalem Mount Scopus, \\ Jerusalem Israel
}

\subsection{Introduction}

In ancient Japan, personal income was measured in terms of a "Koku." One Koku was the amount of rice required to feed one person for a year, about $140 \mathrm{~kg}$ or 13 ounces per day. The income of a "Daimyo" or "great land owner" exceeded $10,000 \mathrm{Koku}$ per year. The great Tokugawa Ieyasu, the first Shôgun of the Edo period (1543-1616), earned annually over four million Koku (Wayland 2003). The Koku system was both a simple and an ingenious measure of income inequality. It was not subject to inflation (only to personal appetite and availability of other food supplements) making it very suitable for both for longitudinal and crosssectional studies. For instance, by comparing regional Koku in years A and A+1, one could estimate that an average person in the central Yamashiro (Kyoto) province, who earned 7 Koku in year A and 7.7 Koku in year A+1, was 10-percent better off than the year before and twice as more affluent as his fellow citizen in the peripheral Hizen, who had to get by with only 3.5 and 3.8 Koku per year.

However, this system of inequality measurement, though simple and affective for a pair-wise comparison, becomes nearly useless when we need to measure the inequality across more than two units (e.g., between Yamashiro, Hizen and Shimozuke provinces). Fortunately, no one seemed to have been concerned with such comparisons in those days.

The computational problems associated with multi-group comparison of income inequality were noticed (apparently for the first time) by the American economist Max Lorenz. In his seminal paper published in 1905 in the Publications of the American Statistical Association, Lorenz highlighted several drawbacks associated with the comparison of wealth concentration between fixed groups of individuals. In particular, he found that while an increase in the percentage of the middle class is supposed to show the diffusion of wealth, a simple comparison of percent shares of persons in each income group may often lead to the opposite conclusion. For instance, while the upper income group in a particular period may constitute a smaller proportion of the total population, the overall wealth of this group may be far larger compared to another time period under study (ibid. pp. 
210-211). The remedy he suggested was to represent the actual inter-group income distribution as a line, plotting "along one axis cumulated percents of the population from poorest to richest, and along the other the percent of the total wealth held by these percents of the populations" (ibid. p.217). As he notes, "With an unequal distribution, the curves will always begin and end in the same points as with an equal distribution, but they will be bent in the middle; and the rule of interpretation will be, as the bow is bent, concentration [of incomes] increases" (p. 217).

The Italian statistician Corrado Gini moved Lorenz's ideas a step further, suggesting a simple and easy comprehendible measure of inequality known as the Gini coefficient. Graphically, the calculation of this coefficient can be interpreted as follows (Atkinson 1983):

$$
\text { Gini coefficient }=\frac{\text { Area between Lorenz curve and the diagonal }}{\text { Total area under the diagonal }}
$$

Mathematically, the Gini coefficient is calculated as the arithmetic average of the absolute value of differences between all pairs of incomes, divided by the average income (see Table 4.1). ${ }^{1}$ The coefficient takes on values between 0 and 1 , with zero interpreted as perfect equality.

In 1920, the British economist Edward Hugh Dalton (1920) suggested an alternative measure of income inequality $(\delta)$, which he estimated as the ratio between logarithms of the arithmetic $\left(\mathrm{x}_{\mathrm{a}}\right)$ and geometric $\left(\mathrm{x}_{\mathrm{g}}\right)$ means of total incomes:

$$
\delta=\frac{\log x_{a}}{\log x_{g}},
$$

where $x_{a}=\sum_{i=1}^{n} x_{i} / n$ and $x_{g}=\sqrt[n]{\prod_{u=1}^{n} x_{u}}$

( $\mathrm{x}_{\mathrm{i}}=$ total income of group $i, n=$ number of groups under comparison). However, even Dalton himself did not attempt to test the proposed measure empirically due to the fact that the calculation of geometric means was vary laborious, if not impracticable, specifically if the number of individual incomes was large (ibid. p. $351)$.

More recent empirical studies proposed and used a variety of additional inequality measurements, such as the Williamson index, Theil index, Atkinson index, Hoover and Coulter coefficients (Williamson 1965; Sen 1973; Atkinson 1983; Coulter 1987; Yitzhaki and Lerman 1991; Sala-i-Martin 1996; Kluge 1999; WBG 1999). Mathematical formulae for these commonly used inequality measures are given in Table 4.1.

These inequality measures basically fall into two classes: measures of dispersion (e.g., the coefficient of variation and Williamson index), and measures

1 The computation includes the cases where a given income level is compared with itself. 
of entropy. The measures in the latter class are given to the following generic formula:

$$
G E(\alpha)=\frac{1}{\alpha^{2}-\alpha}\left[\frac{1}{n} \sum_{i=1}^{n}\left(\frac{y_{i}}{\bar{y}}\right)^{\alpha}-1\right],
$$

where $\mathrm{n}$ is the number of individuals (groups) in the sample, $\mathrm{y}_{\mathrm{i}}$ is the income of individual $i ; \bar{y}$ is the arithmetic mean of individual incomes, and parameter $\alpha$ represents the weight given to differences between incomes at different parts of the income distribution [low values of this parameter make the inequality measure more sensitive to changes in the lower tail of the parameter distribution, while high values make it more sensitive to changes in its upper tail]. The values of GE range from 0 to $\infty$, with zero representing the absolutely even distribution of incomes (WBG 1999).

Table 4.1. Commonly used measurements of regional inequality

Coefficient of variation (CV) (unweighted)

$C V=\frac{1}{\bar{y}}\left[\frac{1}{n} \sum_{i=1}^{n}\left(y_{i}-\bar{y}\right)^{2}\right]^{1 / 2}$

Theil index $(\mathrm{TE}(0))$

$$
T E(0)=\frac{1}{n} \sum_{u=1}^{n} \ln \frac{\bar{y}}{y_{i}}
$$

$$
\begin{gathered}
\text { Hoover coefficient (HC) } \\
H C=\frac{1}{2} \sum_{i=1}^{n}\left|\frac{A_{i}}{A_{t o t}} \frac{y_{i}}{\bar{y}}-\frac{A_{i}}{A_{t o t}}\right|
\end{gathered}
$$

\section{$\underline{\text { Gini (U) (unweighted) }}$}

$$
\text { Gini }=\frac{1}{2 n^{2} \bar{y}} \sum_{i=1}^{n} \sum_{j=1}^{n}\left|y_{i}-y_{j}\right|
$$

Population weighted coefficient of variation (Williamson index (WI))

$$
W I=\frac{1}{\bar{y}}\left[\sum_{i=1}^{n}\left(y_{i}-\bar{y}\right)^{2} \frac{A_{i}}{A_{t o t}}\right]^{1 / 2}
$$

\section{Atkinson index (AT)}

$$
A T=1-\left[\frac{1}{n} \sum_{i=1}^{n}\left[\frac{y_{i}}{\bar{y}}\right]^{1-\varepsilon}\right]^{1 /(1-\varepsilon)}
$$

\section{Coulter coefficient (CC)}

$$
C C=\left[\frac{1}{2} \sum_{i=1}^{n}\left(\frac{A_{i}}{A_{t o t}} \frac{y_{i}}{\bar{y}}-\frac{A_{i}}{A_{t o t}}\right)^{2}\right]^{1 / 2}
$$

Gini (W) (population weighted)

$$
\text { Gini }=\frac{1}{2 \bar{y}} \sum_{i=1}^{n} \sum_{j=1}^{n} \frac{A_{i}}{A_{\text {tot }}} \frac{A_{j}}{A_{\text {tot }}}\left|y_{i}-y_{j}\right|
$$

Note: $\mathrm{A}_{\mathrm{i}}$ and $\mathrm{A}_{\mathrm{j}}=$ number of individuals in regions $i$ and $j$ respectively (regional populations), $\mathrm{A}_{\text {tot }}=$ the national population; $\mathrm{y}_{\mathrm{i}}$ and $\mathrm{y}_{\mathrm{j}}=$ per capita development parameters observed respectively in region $i$ and region $j$ (e.g., per capita income); $\bar{y}$ is the national average (e.g. per capita national income); $\mathrm{n}=$ overall number of regions; $\varepsilon$ is an inequality aversion parameter, $0<\varepsilon<\infty$ [the higher the value of $\varepsilon$, the more society is concerned about inequality). In the literature on inequality measurements, formulas for inequality indexes often differ by a factor of 2 or $1 / 2$. We scale all the indexes between 0 and 1 , to facilitate the interpretation of results.

Compiled from: Sen (1973); Coulter (1987); Kluge (1999); WBG (2001). 
Since the inequality indices in Table 4.1 are abstract mathematical formulae, one can assume that they can be applied to both large and small countries alike. Is this assumption correct? It is well known that the use of different measurement indices in regional analyses gives rise to highly variable results. For example, the notion of optimal regional convergence (i.e. that point where regional convergence also reduces overall nation-level inequality) has been shown to be highly dependent on type of inequality index used (Persky and Tam 1985) as is the measurement of regional price convergence (Wojan and Maung 1998). But does the number, size and rank of regions, also play a part? In this chapter, we shall attempt to answer these questions, using a number of empirical tests. The aim of these tests is to determine whether commonly used inequality measures produce meaningful estimates when applied to a small country.

The chapter is organized as follows. It begins with a brief outline of general principles, which should govern, in our view, the selection of robust inequality measures, followed by an analysis of characteristic features of small countries, which may influence the choice of inequality indices. Then we move to testing the compliance of different commonly used inequality measures against the set of criteria that should characterize, in our view, a robust inequality measure. The tests are run in two phases. First, we use a number of pre-designed distributions, to verify whether a particular inequality measure meets our intuitive expectations concerning inequality estimates. Then, in the second stage of the analysis, we run more formal permutation tests to verify whether different inequality measurements respond sensibly to changes in the population distribution across the space.

\subsection{General Requirements for a Robust Inequality Measure}

As Dalton (1920) noted, many inequality measures, though having intuitive or mathematical appeal, react to changes in income distribution in an unexpected fashion. For instance, if all incomes are simply doubled, the variance quadruples the estimates of income inequality. Dalton's second observation was that some inequality measures do not comply with a basic principle of population welfare set forward by Arthur Pigou in 1912. This is commonly referred to as the principle of transfers and is formulated by Dalton as follows: "if there is only two incomereceivers, and a transfer of income takes place from the richer to the poorer, inequality is diminished" (ibid. p. 351). After applying this principle to various inequality measures, Dalton found that most measures of deviation (e.g., the mean standard deviation from the arithmetic mean, and the coefficient of variation) are perfectly sensitive to transfers and pass the "test with distinction" (ibid. p. 352). The Gini index was found by Dalton sufficiently sensitive to income transfers. He also found that the standard deviation is sensitive to transfers among the rich, while the standard deviation of logarithms is less sensitive to transfers among the rich than to transfers among the poor but still changes when a transfer among the rich takes place. 
Two other fundamental requirements for a "robust measure" of inequality, proposed by Dalton, are the principle of proportional addition to incomes, and the principle of proportional increase in population. According to the former, a proportional rise in all incomes diminishes inequality, while a proportional drop in all incomes increases it. According to the latter principle, termed by Dalton the "principle of proportional additions to persons," a robust inequality measure should be invariant to proportional increases in the population sizes of individual income groups. Dalton's calculations showed that most commonly used measures of inequality comply with these basic principles. Only the most "simple" measures, such as absolute mean deviation, absolute standard deviations and absolute mean difference, fail to indicate any change, when proportional additions to the numbers of persons in individual income groups are applied (ibid. pp.355357, see also Champernowne and Cowell 1998, pp. 87-112). ${ }^{2}$

Sen $(1973$, p.2) distinguished between "measures that try to catch the extent of inequality in some objective sense ... and ....indices that try to measure inequality in terms of some normative notion of social welfare." He also undertook a systematic attempt to test the sensitivity of different inequality measures to changes in income distribution. The indices he tested included: the range; the relative mean deviation; the variance; the coefficient of variation; the standard deviation of logarithms; the Gini coefficient; Theil's entropy measure; Dalton's coefficient, and Atkinson's index. Most of the tested measures appeared to exhibit substantial flaws. For instance, the range was found to ignore the distribution among the extremes (i.e., upper and lower incomes), whereas the sensitivity of the Gini index was found to depend critically "not on the size of the income levels but on... the rank-order position of the person in the ranking by income level" (ibid, $p$. 32). He also found that while the coefficient of variation appears to be sensitive to transfers across all income groups, the standard deviation and the standard deviation of logarithms appeared to be sensitive primarily to transfers in the lower income brackets, and insensitive to transfers among the rich.

In a more recent paper, Yitzhaki and Lerman (1991) note another deficiency inherent to most inequality measures, viz. insensitivity to the position which a specific population subgroup occupies within an overall distribution. Their Gini decomposition technique takes group-specific positions into account. They suggest weighting subgroups by the average rank of their members in the distribution. This is in contrast to the weighting system used more conventionally in which between group inequality is weighted by the rank of the average (Pyatt 1976; Silber 1989). This latter system results in a large residual when inequality is decomposed into within and between groups. In contrast, the Yitzhaki approach results in a more accurate decomposition with no residual (Yitzhaki 1994).

The question of weighting is, of course, intricately connected to the issue of country and regional size. The standard Barro-type growth regressions that look at

2 Dalton (1920, p. 352) distinguishes between measures of relative dispersion and measures of absolute dispersion. Whereas the former measures are dimensionless, the measures of absolute dispersion are estimated in units of income. The latter measures are easily transformed in the former by normalization. 
regional disparities over time, do not weight for regional or country size. This is because regions and countries are treated as individuals and not as groups. No compensation is given for small size just as large people and small people are not given any compensation when looking at a population income distribution (The case could be made that large people "need" more income due to their size and therefore deserve to be compensated). However, the growth regression approach assumes that compensating for population size is tantamount to obscuring the unique identity of places, one of which is their size difference.

Whether regions are individuals or groups is of course a moot (philosophical) point. In contrast to the neo-classical growth regressions approach, the "inequality indices" (Gini) approach seems to accept the fact that countries and regions are groups. The question thus revolves around a suitable weighting system in which the conventional approach (Pyatt 1976; Silber 1989) is pitched against the alternative approach (Yitzhaki and Lerman 1991; Yitzhaki 1994).

\subsection{Characteristic Features of a Small Country That May Affect Inequality Estimates}

The aim of the present inquiry is not to test the conformity of commonly used inequality measures with basic inequality criteria (e.g., principles of transfer, proportional addition to incomes, and proportional addition to population). This task has been accomplished par excellence in previous studies, whose findings we have no reason to doubt. Instead, we shall focus our attention on the features which a robust inequality measure should possess in order to make it fully applicable to a small country, which is the main focus of this volume. First, however, let us outline some essential characteristics of such a country that may affect inequality estimates, at least in theory. Since most of these features were discussed in earlier (frame-setting) chapters (Chapters 1 and 2), we shall outline them only briefly, focusing mainly on the empirical aspects of their measurement.

First, as previously noted, a small country is likely to have a smaller number of regions than a large and more populous nation. Thus, for instance, Japan with its 130-million strong population has 47 regional subdivisions (prefectures), while Israel (6.5 million residents) is split into only six administrative districts (mahozot, in Hebrew). Similarly, Finland (5.2 million residents) is composed by only six provinces (laanit, in Finnish), whereas France (60 million residents) is divided into 22 regions, which are further subdivided into 96 departments (CIA 2003). Although districts and provinces of a small country may further be subdivided into sub-districts and counties, the overall number of such administrative subdivisions in a small country is naturally smaller than the overall number of administrative subdivisions of comparable size in a more populous nation.

The second feature of a small country, which may be important for our analysis, is the varying population sizes of the regions. Although a large country may also have regions of different population sizes, such variation is especially characteristic for a small country, which can be highly mono-centric with a clearly 
emphasized urban core. Due to the geographic concentration of its population, the population size of the core region in a small country may greatly surpass the population of its sparsely populated peripheral regions. For example in Slovenia, the Central Slovenia region containing Ljubljana has over 26 percent of the country's population and the smallest region (Zasavska) has a population one twelfth its size. Similarly in Ireland, the Dublin and Mid East region contains nearly 40 percent of the Irish population and has over seven times the population of the Midland Area. In Finland, the Helsinki metropolitan area dominates the Finnish regional population distribution accounting for nearly 20 percent of national population.

Lastly, regions in a small country may be a subject to rapid change. For instance, economic growth may spread rapidly across neighbouring regions in a small country, reflecting "growth spillover" (Baumont et al. 2000; Carrington 2003). In contrast, in a large and polycentric country, regional growth may be more localized and slow-acting. For instance, we may recall the rapid regional growth attributed to the development of computer-related industries in Ireland in the late 1980s (Roper 2001). The long-term impact of mass immigration to Israel in 1989-1991 is another example of a rapid regional change in a small country. During this period, nearly 600,000 new immigrants arrived, increasing the existing population of the country by some 15 percent. Eventually many newcomers settled in the country's peripheral areas, the Northern and Southern districts, whose populations nearly doubled within a short period of some 3-4 years, boosting the emergence of new major population centres (e.g. Be'er Sheva and Ashdod) and causing considerable changes in the existing urban hierarchy (Lipshitz 1998).

Taking account of these peculiarities, we can introduce the following three basic requirements to a robust inequality measure which should make it applicable to a small country - the subdivision principle; tolerance to size difference, and rank-order insensitivity. These requirements are now outlined:

- Subdivision principle: No matter into how many regions (subdivisions) a country is split, inequality estimates should not change, unless the parameter distribution alters. This requirement is basically in line with Dalton's principle of population, according to which neither replication of population nor merging identical distributions should alter inequality.

- Tolerance to size differences: A robust inequality measure should produce identical estimates for both geographically even and geographically skewed population distributions, providing that the parameter distribution (e.g., distribution of incomes) remains unchanged. For instance, most residents of a country may be concentrated in a single region or they may be dispersed evenly across 10 districts into which the country is split. As long as the income distribution stays the same, regional inequality should not alter.

- Rank-order insensitivity. The inequality estimate should not alter as a result of a change in the sequence in which regions are introduced into the calculation, e.g. ranked either by population size or in alphabetical order. Since regions in a small country may be a subject to rapid changes, both in terms of their population sizes and parameter distributions, compliance with 
this principle will ensure that inequality estimates do not alter simply as a result of changing the position of a region in the rank-order hierarchy.

In order to verify the compliance of commonly used measures of regional inequality with the above requirements, the analysis will be carried out in two stages: pre-designed sensitivity tests (Section 4.4) and random permutation tests (Section 4.5).

\subsection{Pre-designed Sensitivity Tests}

The following specific questions need to be answered:

1. Is an inequality measure sensitive to the overall number of intra-country divisions (regions) covered by analysis?

2. Is an inequality measure sensitive to differences in the population sizes of regions?

3. Does a particular inequality measure respond to changes in the rank-order in which individual regions are introduced into the calculation?

Eight commonly used inequality measures (see Table 4.1) are tested here. The tests are designed as follows. First, we introduce the "reference" distribution (Table 4.2: "Reference distribution"). As Table 4.2 shows, this distribution has 16 internal divisions (regions). The average per capita income in its four central regions doubles that in the 12 peripheral regions - 20,000 and 10,000 Income Units (IUs), respectively. Let us call the former group of regions " $\mathrm{H}$ [igh-income]regions," while 12 other regions will conditionally be termed "L[ow-income]regions."

As the table shows, in the reference distribution, the population is distributed evenly: there are 10,000 residents in each regional cell (see Table 4.2). The total population of the reference system is 160,000 residents and the average income is 12,500 IUs per capita.

\subsubsection{Test 1 - Small Number of Regions}

This test checks whether the overall number of regions matters. To this end, we reduce the overall number of regions to eight, from sixteen in the reference distribution. Total population for this distribution is 80,000 residents, while the average income remains the same and equals 12,500 IUs. Since there are no cardinal changes in income or population distribution, robust inequality indices should indicate the same level of inequality for both the reference and Test 1 distributions (see Table 4.2). 


\subsubsection{Test 2 - Uneven Population Distribution}

This test is designed to trace the response of different inequality measures to regional distribution of population: evenly spread population in the reference distribution vs. unevenly spread population in the Test 2 distribution. Compared to the reference distribution, there are no changes in per capita incomes; only the pattern of population distribution is altered. In particular, the populations of the four central (H-regions) increased to $100,000(4 \times 25,000)$ residents, while the populations of surrounding L-regions shrunk to $60,000(5,000 \times 12)$ residents (see Table 4.2). The total population in this distribution is 160,000 residents and the average income is 16,250 IUs. Since the percent share of population concentrated in four H-regions increased to 62.5 percent $[100,000 \times 100 / 160,000$ (total population $)=62.5 \%]$ from 25 percent in the reference distribution $[40,000 \times 100 /$ $160,000=25 \%$; see Table 4.2$]$, the regional inequality of per capita incomes should expectedly decline.

Table 4.2. The reference and test distributions

\begin{tabular}{|c|c|c|c|c|c|c|c|}
\hline \multicolumn{4}{|c|}{ Reference distribution } & \multicolumn{4}{|c|}{ Test 1 (Number of regions) } \\
\hline \multicolumn{4}{|c|}{ Average income } & \multicolumn{4}{|c|}{ Average income } \\
\hline 10,000 & 10,000 & 10,000 & 10,000 & 10,000 & 10,000 & & \\
\hline 10,000 & 20,000 & 20,000 & 10,000 & 10,000 & 20,000 & & \\
\hline 10,000 & 20,000 & 20,000 & 10,000 & 10,000 & 20,000 & & \\
\hline 10,000 & 10,000 & 10,000 & 10,000 & 10,000 & 10,000 & & \\
\hline \multicolumn{4}{|c|}{ Population size } & \multicolumn{4}{|c|}{ Population size } \\
\hline 10,000 & 10,000 & 10,000 & 10,000 & 10,000 & 10,000 & & \\
\hline 10,000 & 10,000 & 10,000 & 10,000 & 10,000 & 10,000 & & \\
\hline 10,000 & 10,000 & 10,000 & 10,000 & 10,000 & 10,000 & & \\
\hline 10,000 & 10,000 & 10,000 & 10,000 & 10,000 & 10,000 & & \\
\hline \multicolumn{4}{|c|}{ Test 2 (Population distribution) } & \multicolumn{4}{|c|}{ Test 3 (District ranking) } \\
\hline \multicolumn{4}{|c|}{ Average income } & \multicolumn{4}{|c|}{ Average income } \\
\hline 10,000 & 10,000 & 10,000 & 10,000 & 20,000 & 10,000 & 10,000 & 20,000 \\
\hline 10,000 & 20,000 & 20,000 & 10,000 & 10,000 & 10,000 & 10,000 & 10,000 \\
\hline 10,000 & 20,000 & 20,000 & 10,000 & 10,000 & 10,000 & 10,000 & 10,000 \\
\hline 10,000 & 10,000 & 10,000 & 10,000 & 20,000 & 10,000 & 10,000 & 20,000 \\
\hline \multicolumn{4}{|c|}{ Population size } & \multicolumn{4}{|c|}{ Population size } \\
\hline 5,000 & 5,000 & 5,000 & 5,000 & 10,000 & 10,000 & 10,000 & 10,000 \\
\hline 5,000 & 25,000 & 25,000 & 5,000 & 10,000 & 10,000 & 10,000 & 10,000 \\
\hline 5,000 & 25,000 & 25,000 & 5,000 & 10,000 & 10,000 & 10,000 & 10,000 \\
\hline 5,000 & 5,000 & 5,000 & 5,000 & 10,000 & 10,000 & 10,000 & 10,000 \\
\hline
\end{tabular}




\subsubsection{Test 3 - Rank-Order Change}

Our last test is designed to verify whether the sequence in which regions are introduced in the calculation matters. Compared to the reference distribution, there is no change in either the total number of residents $(160,000)$ or in the average per capita income (12,500 IUs). The only change is the location of H-regions: if in the reference distribution these regions are located in the centre of the grid $(6,7,10$ and 11 sequence numbers), in the Test 3 distribution, they are moved to the corners of the grid (1, 4, 13 and 16 sequence numbers - see Table 4.2). Since the percent share of population concentrated in the H-regions has not changed $[40,000 \times 100 / 160,000=25 \%]$, no change in inequality should occur.

Table 4.3. Results of sensitivity tests

\begin{tabular}{ccccc}
\hline $\begin{array}{c}\text { Inequality } \\
\text { index }\end{array}$ & $\begin{array}{c}\text { Reference } \\
\text { distribution }\end{array}$ & $\begin{array}{c}\text { Test 1 } \\
\text { (Number of } \\
\text { regions) }\end{array}$ & $\begin{array}{c}\text { Test 2 } \\
\text { (Population } \\
\text { distribution) }\end{array}$ & $\begin{array}{c}\text { Test 3 } \\
\text { (District } \\
\text { ranking) }\end{array}$ \\
\hline CV & 0.346 & 0.346 & 0.353 & 0.346 \\
WI & 0.346 & 0.346 & 0.298 & 0.346 \\
TE & 0.022 & 0.022 & 0.136 & 0.022 \\
AT & 0.026 & 0.026 & 0.251 & 0.026 \\
HC & 0.150 & 0.150 & 0.144 & 0.150 \\
CC & 0.061 & 0.087 & 0.059 & 0.061 \\
Gini (U) & 0.150 & 0.150 & 0.115 & 0.150 \\
Gini (W) & 0.150 & 0.150 & 0.144 & 0.150 \\
\hline
\end{tabular}

\subsubsection{Sensitivity Test Results}

The results of the tests are reported in Table 4.3 and discussed below.

Test 1: Somewhat surprisingly, despite the unchanged distributions of incomes and populations, $\mathrm{CC}$ indicates a rise in inequality! The use of this index for small countries, with a small number of internal divisions (regions), may thus be misleading, specifically when a comparison with countries of larger sizes is planned.

Test 2: While the five indices (WI, CC, HC, Gini (U) and Gini (W)) indeed indicate a drop in regional inequality compared to the reference distribution, three other measures (CV, TE and AT) indicate an increase (!) in income disparity. Characteristically, Gini (W) indicates only a marginal drop in inequality (from 0.150 in the ref. distribution to 0.144 in the Test 2 distribution) despite a considerable increase in the population share of H-regions. The use of CV, TE, AT, and Gini (W) for small countries (which are often characterized by extremely uneven regional distributions of population) may thus lead to erroneous results.

Test 3: The test indicates no performance problems with any of the indices tested. Numerically, the results of the test appear to be identical to those obtained for the ref. distribution (see Table 4.3). 


\subsection{Permutation Tests}

For more formal sensitivity testing of inequality measures, we used the statistical technique known as bootstrapping (Hesterberg et al. 2002). Traditional methods of calculating parameters for a given statistic (e.g., a certain measure of inequality) are based upon the assumption that the statistic is asymptotically normally distributed and use known transformations for parameter calculation. However, re-sampling techniques, such as bootstrapping, provide estimates of the standard error, confidence intervals, and distributions for any statistic by testing it directly against a large number of randomly drawn re-samples. 1000 re-samples are considered as a minimal number recommended for estimating parameters of a statistic, whereas larger numbers of re-runs increase the accuracy of estimates.

In particular, we ran two separate tests, as described below:

- Test 1 (Unrestricted test): The distribution of income was set identical to the reference distribution (see Table 4.2) and the average income was kept constant (12,500 IUs). Concurrently, the population was distributed across 16 regional cells at random and was allowed to vary slightly around the average population total, which was not restricted a-priori.

- Test 2 (Restricted test): The income distribution, the average income, and the total population of the system were kept constant and identical to the reference distribution (see Table 4.2). In order to comply with these restrictions, the population was redistributed within the H-regions and Lregions, without allowing population exchanges between these two groups of regions.

For each test, 1000 permutations (re-samples) were run. For the sake of clarity and brevity and to avoid overloading the reader with unnecessary technical details, we discuss below only those results that appear to exhibit most characteristic trends.

\subsubsection{Unrestricted Test}

The results of the re-sampling for five inequality indices - CV, Gini (U), AT, $\mathrm{TE}(0)$, and WI are reported in Figure 4.1. While CV, Gini (U), AT and TE(0) appear to exhibit the response pattern shown in Figure 4.1A, the rest of the indices tested (that is, WI, CC, HC and Gini (W)) exhibit the response pattern diagrammed in Figure 4.1B. The conclusion is thus straightforward: the former group of indices is not sensitive to the variation in population distribution across regional cells. They may thus lead to spurious results when used for small countries, which are often characterized by rapid changes in population patterns, due to (inter alia) the impact of immigration. 


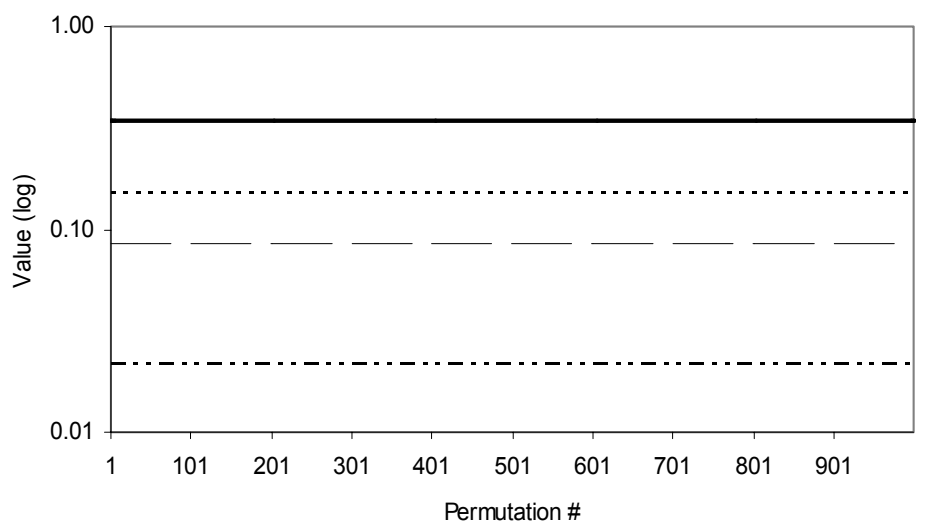

A

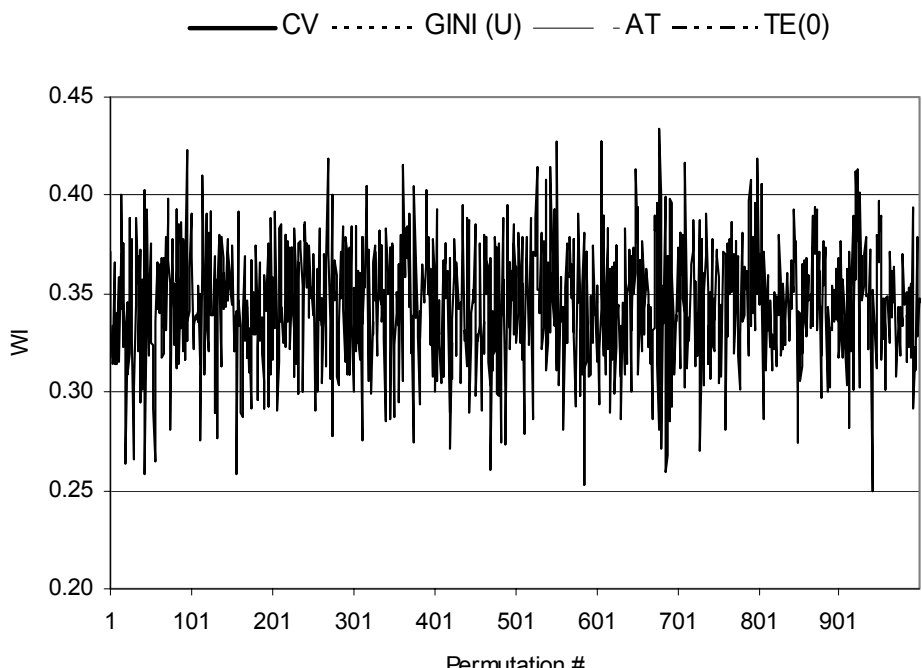

B

Fig. 4.1. Results of permutation tests (Test 1: unrestricted test) for selected inequality measures - CV, Gini (U), AT and TE(0) (A) and WI (B)

Note: see text for explanations.

\subsubsection{Restricted Test}

When population movements are restricted (i.e., the population is allowed to circulate only within the H-regions and within the L-regions, without direct population exchanges between the two), only the $\mathrm{CC}$ index appears to respond to population re-sampling, exhibiting the oscillation response pattern (see Figure 


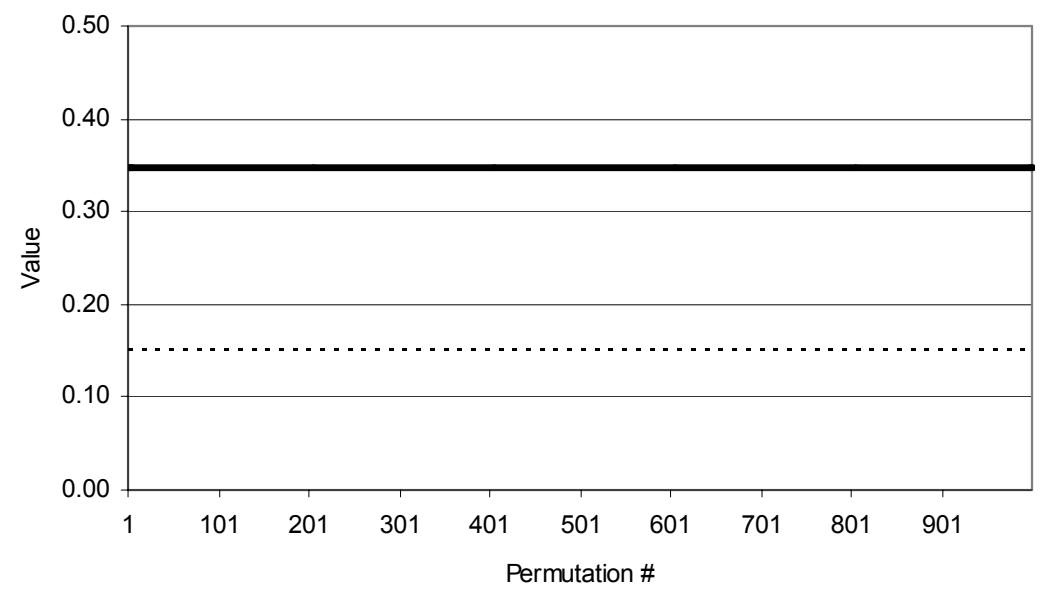

A GINI (U)

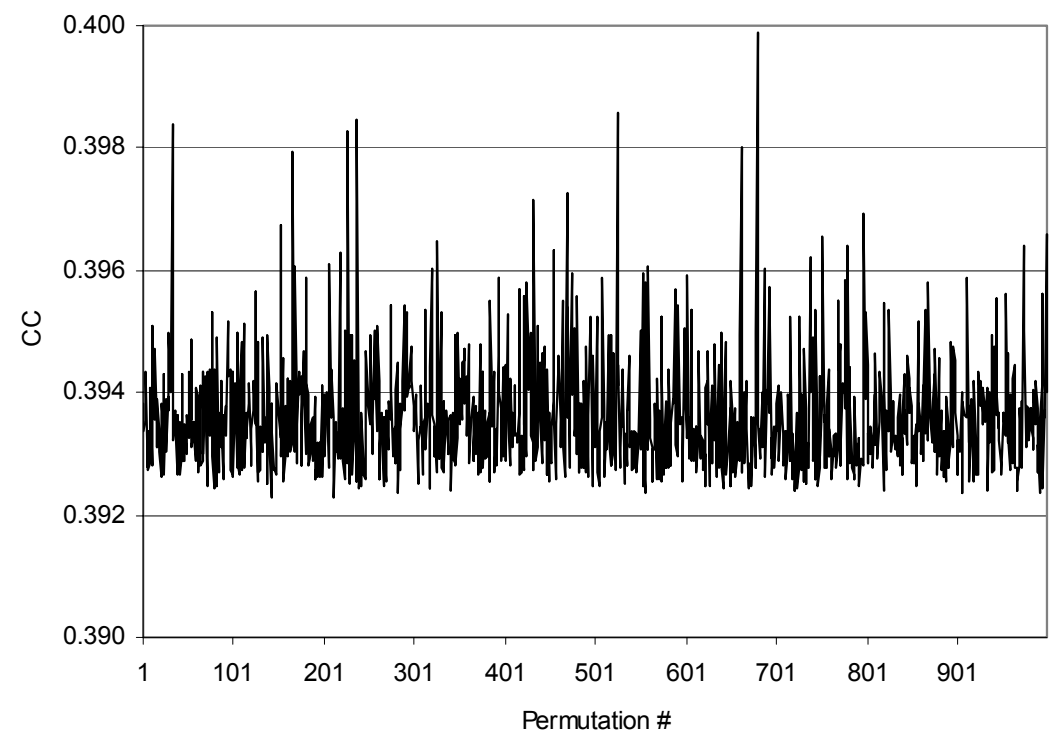

B

Fig. 4.2. Results of permutation tests (Test 2: restricted test) for selected inequality measures - WI and Gini (U) (A) and CC (B)

Note: see text for explanations.

4.2B), whereas all other indices tested (i.e., CV, WI, HC, Gini (U), Gini (W), AT and $\operatorname{TE}(0)$ ) fail to respond to changes in the population distribution across the regional cells (see Figure 4.2A). However, such a situation (in which population movements are geographically restricted) may be considered rather unlikely 
(specifically for open economies) and thus a failure of an inequality measure to pass this test may be considered only as a minor performance flaw.

\subsection{Conclusions}

Though individual studies of regional disparity may deal with separate development measures - population growth, wages, welfare, regional productivity, etc. the use of an integrated indicator is often essential, particularly if a comparative (cross-country) analysis is required. In order to measure the extent of disparities, various indices of inequality are commonly used. These indices may be classified into two separate groups (Kluge 1999):

- Measures of deprivation (Atkinson index, Theil redundancy index, Demand and Reserve coefficient, Kullback-Leibler redundancy index, Hoover and Coulter coefficients, and the Gini index);

- Measures of variation, such as the coefficient of variation and Williamson's index.

In this chapter, we did not attempt to assess whether these measurements reflect either the "true meaning" or "underlying causes" of regional inequality. Neither did we try to establish whether geographic inequality is a positive socio-economic phenomenon or a negative one. We shall leave these philosophical questions for other studies. Our task was simple: we attempted to determine whether commonly used inequality measures produce meaningful estimates when applied to small countries, thus making it possible to compare the results of analysis obtained for such countries with those obtained elsewhere.

As we argue, a small country may differ from a country of larger size in three fundamental features. First, it is likely to have a relatively small number of regional divisions. Second, its regional divisions are likely to vary considerably in their population sizes. Lastly, regions of a small country may rapidly change rankorder positions in the country-wide hierarchy, by changing their attributes (e.g., population and incomes). In contrast, in a large country such rank-order changes may be both less pronounced and slower-acting.

In order to formalize these distinctions, we designed a number of simple empirical tests, in which income and population distributions, presumably characteristic for small countries, were compared with a "reference" distribution, assumed to more accurately represent a country of a larger size. In the latter (reference) distribution, the population was distributed evenly across regional divisions and assumed to be static.

In the first test, we checked whether the overall number of regions matters. In the second, we tested whether different inequality indices respond to differences in the regional distribution of population, viz., evenly spread population in the reference distribution vs. unevenly spread population in the test distribution. Finally, in the third test, we verified whether different inequality indices were sensitive to the sequence in which regions are introduced into the calculation. 
Somewhat surprisingly, none of the indices we tested appeared to pass all the tests, meaning that they may produce (at least theoretically) misleading estimates if used for small countries. However, two indices - WI and Gini (W) - appeared to exhibit only minor flaws and may thus be considered as more or less reliable regional inequality measures.

Although further studies on the performance of different inequality indices may be needed to verify the generality of our observations, the present analysis clearly cautions against indiscriminate use of inequality indices for regional analysis and comparison.

\section{Acknowledgement}

Our gratitude is due to Jacques Silber for helpful comments on an earlier draft. We are also indebted to Alex Portnov for designing an algorithm for the permutation tests and to Goetz Kluge for verification of the calculation results.

\section{References}

Atkinson AB (1983) The economics of inequality (2nd Edition). Clarendon Press, Oxford Baumont C, Ertur C, Le Gallo J (2000) Geographic spillover and growth: a spatial econometric analysis for European regions. Paper presented at the 6th RSAI World Congress 2000 "Regional Science in a Small World", Lugano, Switzerland, May 1620,2000

Carrington A (2003) A divided Europe? Regional convergence and neighbourhood spillover effects. Kyklos 56:381-393

Champernowne DG, Cowell FA (1998) Economic inequality and income distribution. Cambridge University Press, Cambridge, UK

CIA (2003) 2002 World factbook. Central Intelligence Agency, Washington, D.C. (Internet edition)

Coulter P (1987) Measuring unintended distributional effects of bureaucratic decision rules. In: Busson T, Coulter P (eds) Policy evaluation for local government. Greenwood Press, New York

Dalton H (1920) The measurement of the inequality of incomes. The Economic Journal 30(199):348-361

Hesterberg T, Monaghan S, Moore DS, Clipson A, Epstein R (2002) Bootstrap methods and permutation tests, Ch18. In: Moore DS, McCabe GP, Duckworth WM, Sclove SL (eds), The practice of business statistics. WH Freeman and Co., NY 18:4-25

Kluge G (1999) Wealth and people: inequality measures (Internet edition)

Lipshitz G (1998) Country on the move: migration to and within Israel, 1948-1995. Kluwer Academic Publishers, Dordrecht

Lorenz MO (1905) Methods of measuring the concentration of wealth, Publications of the American Statistical Association 9(70):209-219

Persky JJ, Tam M-YS (1985) The optimal convergence of regional incomes. Journal of Regional Science 25(3):337-351

Pigou AC (1920; 1938 reprint) The economics of welfare. MacMillan and Co, London 
Pyatt G (1976) On the interpretation of disaggregation of the Gini coefficient. Economic Journal 86:243-255

Roper S (2001) Innovation policy in Ireland, Israel and the UK: evolution and Success. In: Felsenstein D, McQuaid R, McCann P, Shefer D (eds) Public investment and regional economic development. Edward Elgar, Cheltenham, UK, 75-91

Sala-i-Martin X (1996) Regional cohesion: evidence and theories of regional growth and convergence. European Economic Review 40:1325-1352

Sen A (1973) On economic inequality (The Radcliffe lectures series). Clarendon Press, Oxford

Silber J (1989) Factor components, population subgroups and the computation of the Gini index of Inequality. The Review of Economics and Statistics 71(2),107-115

Wayland D (2003) Measure it in Japanese (http://www.thefightschool.demon.co.uk)

WBG (2001) Inequality measurement. World Bank Group, Washington, D.C. (Internet edition)

Williamson JG (1965; 1975 reprint) Regional inequalities and the process of national development: a description of the patterns. In: Friedmann J, Alonso W (eds), Regional policy. The MIT Press, Cambridge, Massachusetts 158-200

Wojan TR, Maung AC (1998) The debate over state-level inequality: transparent method, rules of evidence and empirical power. The Review of Regional Studies 28(1),63-80

Yitzhaki S (1994) Economic distance and overlapping distributions. Journal of Econometrics 61,147-159

Yitzhaki S, Lerman RI (1991) Income stratification and income inequality. Review of Income and Wealth 37(3):313-329 
